\title{
MELHORIA DA ATENÇÃO À SAÚDE DO IDOSO NO ESF DR. GARIBALDI CARRERA MACHADO
}

\author{
IMPROVEMENT OF THE ELDERLY HEALTH CARE IN THE ESF DR. \\ GARIBALDI CARRERA MACHADO
}

\author{
Diego Germán Ledesma ${ }^{1}$, Layza Kowalski², Tiago Bittencourt Oliveira² \\ ${ }^{1}$ Secretaria Municipal de Saúde do Município de Santo Ângelo, RS, Brasil; ${ }^{2}$ Universidade \\ Regional Integrada do Alto Uruguai e das Missões (URI), Santo Ângelo, RS, Brasil.
}

\section{RESUMO}

Objetivo: realizar cobertura ao idoso na Estratégia Saúde da Família (ESF) Dr. Garibaldi Carrera Machado, assim como ampliar o acompanhamento médico e odontológico dos pacientes atendidos nesta área. Metodologia: estudo descritivo na modalidade relato de experiência. A descrição dos dados foi realizada com base nos relatos e dados obtidos no trabalho de especializaçáo em Saúde da Família. Resultados: Estima-se que o território apresenta 300 pessoas idosas, representando $10 \%$ da população total do local, sendo esta de 3.000 habitantes. A intervenção teve com foco a população idosa de áreas do território com cobertura de agentes comunitários de saúde e nas quais foi possível fazer visitas domiciliares. De todos os idosos, 154 fizeram parte da intervençáo, alcançando no final de 3 meses uma cobertura de 51,3\%. Os pacientes tiveram suas cadernetas de idosos, exames, consultas odontológicas atualizadas, também participaram de orientaçóes e açôes de promoção da saúde como alimentação saudável, prática de exercícios físicos, cessação do tabagismo. Alguns idosos foram encaminhados para médicos especialistas ou para programas da secretaria municipal de Saúde como o combate ao tabagismo. Também, foi importantíssimo as parcerias com as universidades locais e escola do bairro para apoio e suporte nas atividades. Conclusáo: A comunidade teve boa aceitação e participaçáo no programa sobre saúde do idoso, abrangendo mais de $50 \%$ dos idosos do território. Eles reconheceram o grande esforço que a equipe fez, apesar da carência de recursos e infraestrutura.

Descritores: Atenção primária à saúde; Saúde do idoso; Humanização da Assistência; Assistência domiciliar.

\section{ABSTRACT}

Objective: To provide coverage to the elderly in the Family Health Strategy (FHS) Dr. Garibaldi Carrera Machado, as well as expand the medical and dental follow-up of patients treated in this area. Methodology: a descriptive study in the experience report modality. The data description was based on the reports and data obtained from the Family Health specialization work. Results: It was estimated that the territory has 300 elderly people, representing $10 \%$ of the total population of the place, which is 3,000 
inhabitants. The intervention focused on the elderly population of areas of the territory covered by community health agents. It was possible to make home visits. Of all the elderly, 154 were part of the intervention, reaching a coverage of $51.3 \%$ at the end of 3 months. The patients had their elderly records, exams, dental appointments updated, also participated in health promotion guidelines and actions such as healthy eating, physical exercise, and smoking cessation. Some seniors were referred to specialists or secretarial health programs such as tobacco control. Also, it was very important the partnerships with the universities and neighborhood school to support the activities. Conclusion: The community had a good acceptance and participation in the elderly health program, covering more than 50\% of the elderly in the territory. They recognized the great effort the team made despite the lack of resources and infrastructure.

Descriptors: Primary health care; Elderly Health; Humanization of Assistance; Home care.

\section{INTRODUÇÁO}

Segundo a Organização das Nações Unidas (ONU), o número global de pessoas idosas - com 60 ou mais anos de idade, irá aumentar de 962 milhóes em 2017 para 1,4 bilhôes em 2030, e 2,1 bilhões em 2050, quando todas as regiões do mundo terão quase um quarto ou mais de sua população idosa ${ }^{1}$. No Brasil, a expectativa do Instituto Brasileiro de Geografia e Estatística (IBGE) é de que a população com mais de 60 anos de idade seja, aproximadamente, $11 \%$ da população geral até o ano de $2025^{2}$.

É sabido que nessa faixa etária, condições fisiológicas, fisiopatológicas e sociais são alteradas, o que a diferencia das demais faixas etárias, constituindo-se uma camada da população com características e necessidades próprias. Esses indivíduos são mais vulneráveis a doenças degenerativas de começo insidioso, como as cardiovasculares e cerebrovasculares, o câncer, os transtornos mentais e os estados patológicos que afetam o sistema locomotor e os sentidos ${ }^{3}$.

O idoso é vulnerável à exclusão social, por não ter vínculo com o trabalho e com os colegas, pela dificuldade de comunicação com as geraçóes mais jovens, pelo isolamento em relação à família, pela perda de autonomia física e funcional e, ainda, pelas dificuldades $\mathrm{da}$ adaptação às novas tecnologias ${ }^{4}$. Portanto, é significativo que os idosos sejam bem acolhidos nos serviços de saúde, que sejam ouvidos e atendidos em suas demandas, sem deixar de reconhecer suas reais necessidades e limitaçôes. Traçar ações estratégicas para os idosos é um dos grandes desafios a serem enfrentados pelas políticas de Saúde Pública, uma vez que estas açóes dependem de um olhar integral para que possam proporcionar uma melhor qualidade de vida para os idosos ${ }^{5}$. Levando em consideraçáo isto, o presente trabalho foi realizado com enfoque na saúde do idoso, tendo como objetivo realizar cobertura total ao idoso na Estratégia Saúde da Família (ESF) Dr. Garibaldi Carrera Machado, assim como ampliar o acompanhamento médico e odontológico dos pacientes atendidos nesta área, mantendo a qualidade do atendimento e serviços ofertados. 


\section{MÉTODO}

Trata-se de um estudo descritivo na modalidade relato de experiência. A vivência foi desenvolvida na Estratégia Saúde da Família (ESF) Dr. Garibaldi Carrera Machado, a qual abrange o bairro Indubras, no município de Santo Ângelo, noroeste do estado do Rio Grande do Sul. Segundo estimativas da secretaria de saúde do município, o território apresenta 300 pessoas idosas, representando $10 \%$ da populaçáo total do local, sendo esta de 3.000 habitantes.

\section{Aspectos Éticos}

O projeto foi aprovado pelo Comitê de Ética em Pesquisa da Universidade Federal de Pelotas, quanto às questóes éticas e metodológicas, de acordo com a resoluçáo 466/2012 do Conselho Nacional de Saúde.

\section{Coleta de Dados}

Os dados do estudo foram coletados em um período de 12 semanas, de março a junho do ano de 2015. Os autores utilizaram como referência os protocolos do Ministério da Saúde (MS), assim como os Cadernos de Atenção Básica relacionados à Saúde do Idoso e Envelhecimento. A equipe da ESF recebeu capacitação sobre os protocolos de saúde do idoso do MS, assim como os Agentes Comunitários de Saúde (ACS) foram orientados para a busca ativa de pacientes idosos.

Para ampliar a oferta aos idosos no local de abrangência do ESF, realizou-se monitoramento semanal de novos usuários e de usuários que consultavam regularmente e não possuíam caderneta do idoso. A comunidade foi devidamente informada sobre importância do acompanhamento periódico do idoso em relação a sua saúde.

Para melhorar a qualidade da atençáo ao paciente idoso no ESF, realizou-se a Avaliação Multidimensional Rápida (AMR) em todos os idosos acompanhados, assim como atentou-se que todos tivessem exames clínicos e laboratoriais de rotina atualizados. Todos pacientes idosos foram rastreados para Diabetes Mellitus (DM) e Hipertensão Arterial Sistêmica (HAS). Foram registrados todos os idosos acamados ou com problemas de locomoção, realizando visitas domiciliares a cada 2 ou 3 semanas, de acordo com o caso. Avaliou-se os idosos cadastrados, em relação a saúde bucal, tanto na necessidade de atendimento, como na qualidade das próteses.

Em relação às açóes destinadas a melhoria da adesão do paciente idoso, a equipe contou com o auxílio da Faculdade de Biomedicina do Instituto Cenecista de Ensino Superior (IESA) para lhes proporcionar o acesso a coleta de exames de sangue, urina e fezes. 
Para a melhoria do registro das informaçóes referentes a população idosa, organizou-se os prontuários dos pacientes, criando novos quando ausentes ou restaurados quando presente.

Para a realização da Avaliação Multidimensional Rápida nos pacientes idosos, incentivou-se a participação dos pacientes e suas famílias em diferentes atividades coletivas e grupais, assim como, foram identificados todos os pacientes idosos que moravam num ambiente de solidão ou de contexto social desfavorável ou de risco, com rede social e familiar precária. Os pacientes com caso de doença pulmonar obstrutiva crônica e tabagistas, foram inseridos no Programa Nacional de Tabagismo (PNT).

Para a promoção à saúde do paciente idoso, a equipe foi capacitada pelo médico autor do trabalho sobre a promoção à saúde e prevenção de doenças, na correta relação médico/paciente e médico/equipe, assim como a importância da anamnese e do tempo mínimo de 15-20 minutos de duração da consulta. Desse modo, todos os pacientes receberam orientações sobre alimentação saudável, atividade física e importância da saúde bucal e cuidado das próteses dentária.

\section{RESULTADOS E DISCUSSÃO}

Dentre a populaçáo estimada de 300 idosos, 154 participaram ativamente do estudo, alcançando-se uma cobertura de 51,3\%. A cobertura do estudo foi de $26 \%$ (78 usuários) no $1^{\circ}$ mês, de 38\% (114 usuários) no 2º mês e, de 51,3\% (154 usuários) no $3^{\circ}$ mês. Sendo assim, alcançou-se mais da metade da população adstrita estimada.

O bairro Indubras, historicamente, se formou por aposentados do parque industrial e das áreas rurais que o rodeiam. Um dos motivos de não alcançar o objetivo de 300 idosos, também se deu pela falta de ACS em áreas do território, o que não permitiu atingir alguns locais e fazer rastreamento e busca ativa de pacientes, à exceção dos idosos de áreas rurais que consultavam espontaneamente na ESF. As consultas aumentaram também por causa da epidemia da Dengue que atingiu a cidade, e as campanhas de vacinação da Gripe. No total o ESF apresenta 6 ACS, das quais 3 estavam de licença no momento do trabalho e, outras 2 tem outros empregos, além de agente comunitário de saúde, o ESF não possui uma técnica enfermagem, o que sobrecarrega a enfermeira e o médico.

A situação atual do Brasil, com grave crise econômica, pode piorar ainda mais a saúde dos idosos, por dificultar a proteção social daqueles mais vulneráveis, que precisam cada vez mais atenção, sobretudo na área da saúde ${ }^{5}$.

A epidemia da Dengue e a Campanha de Vacinação da Gripe, foram fatores que de certa forma também atrapalharam a cobertura do programa por serem situações de emergência e prioridade para os idosos, levando em consideração as mortes acometidas 
na cidade por esses problemas. Os pacientes, ainda hoje, preocupam-se mais pela dengue do que pela HAS ou a DM.

O aumento da cobertura da população e a melhoria da estruturação da Estratégia Saúde da Família (ESF) ainda enfrenta problemas, também em sua atuação, na prática é diferente do proposto pelos documentos do MS, ainda se privilegia o manejo de doenças crônicas com uma visão predominantemente biomédica ${ }^{5}$. Ainda nas ESFs perpassa o "olhar fragmentado", curativista, voltada para soluçóes rápidas e pontuais, o trabalho da equipe do ESF deve garantir uma ruptura com a visão centrada na doença ${ }^{6}$.

Os recursos da secretaria municipal de saúde naquele momento se orientam nas direçôes do combate à dengue e gripe e, muitas vezes, há pouco recurso para materiais nas áreas de atendimento das ESFs. O principal foi a falta de meios de transporte, que auxiliou na redução do nosso objetivo, pela incapacidade de chegar até as áreas mais afastadas do território. A cobertura de uma equipe é muito sensível a falta de recursos, sobretudo humanos (equipe incompleta) e materiais (falta de meios de transporte). A equipe precisa de pelo menos mais 4 ACS e de uma técnica de enfermagem permanente na unidade, para funcionar em sua capacidade máxima. Nesse sentido, os cursos de psicologia e de enfermagem da cidade da Universidade Regional Integrada (URI) forneceram estagiários todo o tempo do estudo, o que ajudam muito no funcionamento do serviço.

Os prontuários foram agrupados por família, caso o idoso morava sozinho, colocou-se seu prontuário no envelope do familiar mais próximo ao domicílio deste, ou em uma família de referência que more perto da casa do paciente. Também, ocorreu distribuição e atualização das cadernetas do idoso, assim como do hipertenso e do diabético, também foram preenchidas fichas espelho, com o auxílio dos acadêmicos de Enfermagem e Psicologia.

Os idosos faltosos receberam busca ativa, e se moram numa área sem ACS, a equipe tentou estabelecer contato via telefone, usando os celulares próprios e quando cada um se encontra na sua casa, devido que no ESF não tem sinal efetivo de celular pois se tratar de uma zona mais afastada da cidade. Em relação à proporção de idosos faltosos às consultas que receberam busca ativa, no $1^{\circ}$ mês foi de 9 usuários, no $2^{\circ}$ mês foi de 11 usuários e, no $3^{\circ}$ mês foi de 1 usuário.

Todos os idosos que realizaram consulta receberam orientação nutricional para hábitos alimentares saudáveis, nos 3 meses (78, 114 e 154 usuários respectivamente), assim como a mesma proporção de idosos que receberam orientação sobre prática regular de atividade física. A perda de força e massa muscular, assim como o desempenho físico tem sido associado com síndrome geriátricas, morbidade e mortalidade em idosos, portanto, uma das melhores opções de tratamento não farmacológico é a prática regular de atividade física. A prática regular de exercício físico reduz os efeitos fisiológicos do processo de envelhecimento e aumenta o tempo de vida ativa, melhorando aspectos 
psicológicos e cognitivos, o que reduz a progressão de doenças crônicas e condições de incapacitantes ${ }^{7}$.

Os idosos que receberam orientação sobre higiene bucal, no $1^{\mathrm{o}}$ mês foi de 98,7\% (77/78 usuários), no $2^{\circ}$ mês foi de 99,1\% (113/114 usuários) e no $3^{\circ}$ mês foi de 99,4\% (153/154 usuários). Os indivíduos idosos com primeira consulta odontológica programada, no $1^{\circ}$ mês foi de $98,7 \%$ (77/78 usuários), no $2^{\circ}$ mês foi de $99,1 \%$ (113/114 usuários) e no $3^{\circ}$ mês foi de 99,4\% (153/154 usuários). Os idosos geralmente apresentam baixos níveis de saúde bucal, tendo como principal problema a perda de dentição, necessitando do uso de próteses para suprir suas necessidades mastigatórias. Ainda a perda de dentes, problemas periodontais originam gengivite, periodontite, gengivoses e, outras consequências, que são alguns dos problemas observados no envelhecimento ${ }^{8}$. Observam-se, ainda, dores na articulação-temporo-mandibular devido às perdas de dentes, próteses antigas com desgaste, próteses mal ajustadas, desgastes dos próprios dentes, com a perda da dimensão vertical. Portanto, a saúde bucal é uma parte integrante da saúde geral e que tem sido colocada no esquecimento, quando se discutem as condiçóes de saúde da população idosa ${ }^{9}$.

A parcela deidosos com Avaliação Multidimensional Rápida em dia foi aumentando no percorrer dos meses, sendo de 93,6\% (73/78 usuários) no $1^{\circ}$ mês, de 93,9\% (107/114 usuários) no $2^{\circ}$ mês, e de 96,8\% (149/154 usuários) no $3^{\circ}$ mês. Quanto ao exame clínico apropriado em dia, no $1^{\circ}$ mês foi de $94,9 \%$ (74/78 usuários), no $2^{\circ}$ mês foi de $94,7 \%$ (108/114 usuários) e no 30 mês foi de 96,1\% (148/154 usuários).

Os indivíduos hipertensos e/ou diabéticos, com solicitação de exames complementares periódicos em dia, no $1^{\circ}$ mês foi de 80,7\% (63/78 usuários), o $2^{\circ}$ mês $86,0 \%$ (98/114 usuários) e o $3^{\circ}$ mês 89,6\% (138/154 usuários). Sobre a priorização de prescrição de medicamentos da Farmácia Popular, no $1^{\circ}$ mês foi de 96,2\% (75/78 usuários), no $2^{\circ}$ mês 95,6\% (109/114 usuários) e no $3^{\circ}$ mês 96,8\% (149/154 usuários). Todos os idosos também foram rastreados quanto a sua pressão arterial e glicemia.

Nos atendimentos se deu prioridade aos medicamentos genéricos contidos na RENAME que podem ser obtidos na própria ESF ou nas Farmácias Municipais e Populares. Uma boa parte dos pacientes foram encaminhados para especialistas, e voltaram para casa com receitas de medicamentos de referência ou nome comercial. Orientou-se, para que os pacientes voltassem na unidade para revisar essas receitas pelo médico para tentar trocar por genérico ou similar equivalente, assim o paciente conseguia seguir o tratamento. Também, auxiliou-se na organização e preenchimentos da documentação necessária para abertura de processo administrativo na Secretaria Municipal de Saúde para solicitação de Medicamentos Especiais, todos as solicitações foram atendidas até o final do período do estudo, especialmente em medicaçóes específicas e especiais, em 
todos os casos. Os pacientes que usavam tabaco e, que na medida se colocavam dispostos a pararem eram encaminhados ao programa de combate ao tabagismo do município.

O Brasil tem buscado implementar, desde a década de 90, políticas que visam o acesso aos medicamentos, sendo um dos poucos países que garante o acesso a medicamentos essenciais e gratuito a todos. Entretanto, tem sido mostrado que o pagamento privado para uso de medicamentos é maior a cada ano no Brasil, o que também reforça que a população de maior renda é que consegue acesso a medicação, seja por recursos próprios

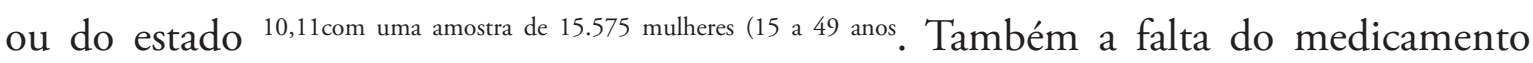
pode gerar o abandono do tratamento, o que pode gerar a piora do estado de saúde e o retorno a atendimento médico ou serviços de saúde. Além disso, a falta de acesso pode comprometer a renda familiar com gastos com saúde ${ }^{12,13}$.

Foram devidamente notificados alguns casos suspeitos de violência familiar contra o idoso, de abandono e também alguns foram encaminhados para acompanhamento no CAPS e CAPS AD por dependência química a álcool e benzodiazepínicos.

No tocante de idosos acamados ou com problemas de locomoção cadastrados, no $1^{\circ}$ mês foi de $21,4 \%$ (6/78 usuários), no $2^{\circ}$ mês foi de $29 \%$ ( $9 / 114$ usuários) e no $3^{\circ}$ mês foi de 35,3\% (12/154 usuários). Todos os idosos acamados receberam visitas domiciliares, a exceção de 1 no primeiro mês.

A visita domiciliária é importante pois junto aos familiares é possível estabelecer um suporte mais adequado às necessidades da pessoa idosa, articulando com os familiares aspectos do cuidado. As visitas domiciliares possibilitam maior vínculo familiar, reunindo seus aspectos afetivos, culturais, religiosos e sociais. O vínculo é um dos princípios da ESF de maior importância. Consiste na construçáo de uma relação de confiança entre o paciente e profissionais de saúde. A construção de vínculo possibilita corresponsabilização pela saúde. Ainda esta ação permite uma melhor compreensão das necessidades de saúde do usuário, fomenta a efetividade das ações assistenciais, favorece a participação ativa do usuário, qualificando assim a assistência oferecida ${ }^{6}$.

Sobre a proporção de idosos com registro na ficha de acompanhamento/espelho em dia, no $1^{\circ}$ mês foi de 92,3\% (72/78 usuários), no $2^{\circ}$ mês foi de 94,7\% (108/114 usuários) e no $3^{\circ}$ mês foi de $97,4 \%$ (150/154 usuários). Já a parcela de idosos com Caderneta de Saúde da Pessoa Idosa, no $1^{\circ}$ mês foi de 97,4\% (76/78 usuários), no $2^{\circ}$ mês foi de $96,5 \%$ (110/114 usuários) e no $3^{\circ}$ mês foi de 97,4\% (150/154 usuários).

A integralidade das açóes na saúde é muito importante, quer na perspectiva de articulação entre açôes de promoção à saúde, preventivas e curativas, ou na visão holística do ser humano biopsicossocial. A integralidade precisa ocupar lugar de destaque na oferta dos cuidados em saúde. Portanto, tais práticas devem ser operacionalizadas no SUS pela Atenção Primária à Saúde (APS) que serve como principal porta de entrada do sistema ${ }^{5}$. 
Apesar das mudanças administrativas municipais terem afetado o funcionamento da unidade, bem como, o repasse de insumos e a disponibilidade de transporte, ao final do estudo a situação foi regularizada juntamente com maior aporte de medicamentos e equipamentos para unidade.

A organização e a educação permanente em saúde são um problema para as açóes dos ESFs e do SUS como um todo em vários lugares do Brasil. Ainda, também deve-se citar que a falta de capacitação dos profissionais pelos gestores, a não inclusão de agendas para idosos e a inexistência de protocolos locais para nortear o cuidado ao idoso em sua integralidade prejudicam muito a qualidade do serviço ofertado ${ }^{6,14}$. Detectado este problema foi realizado pelo médico e enfermeira da equipe palestras quinzenais com a equipe do ESF, sobre a Política Nacional de Humanização em saúde e protocolos sobre saúde do paciente idoso, tendo como fonte o material do Ministério da Saúde ${ }^{15,16}$.

O exame físico dos pacientes sempre foi completo, tanto pela equipe de enfermagem, como na consulta médica, desde o início das atividades e está composta por um acolhimento e uma consulta inicial de enfermagem, uma relação médico/paciente de confiança, com anamnese e exame físico completos, assim como a solicitação de exames complementares pertinentes.

Por regra geral, no momento do diagnóstico, se solicita a todo paciente com DM e HAS, os exames pertinentes. No caso dos idosos, $96,5 \%$ dos pacientes que participaram do programa teve solicitação de exames em dia. O problema da solicitaçáo de exames foi a cota baixa que a SMS oferece. Mas uma parceria com o curso de Biomedicina da cidade conseguiu que os pacientes idosos do programa não dependessem de ditas cotas municipais e que os exames fossem feitos diretamente no laboratório escola da universidade. $\mathrm{O}$ bairro Indubras por ser tão periférico e rural, muitas vezes dificulta que os pacientes "viagem" até a cidade para realizar exames, sobretudo os idosos que moram sozinhos. A parceria com a Faculdade CNEC Santo Ângelo (IESA) se estendeu a coleta de sangue e urina para exames laboratoriais gerais. Os exames foram solicitados pelo médico do ESF que facilitou o acompanhamento e monitoramento dos idosos do estudo.

\section{CONCLUSÃO}

A intervenção tratou sobre a melhoria da Atenção à Saúde do Paciente Idoso (pessoas de 60 ou mais anos) no Bairro INDUBRAS da cidade de Santo Ângelo/RS. Na área de abrangência da unidade existem oficialmente 3000 pessoas cadastradas, das quais $300(10 \%)$ são idosas. A intervenção teve com foco a população de áreas do território com ACS e nas quais foi possível fazer visitas domiciliares. De todos os idosos, 154 fizeram parte da intervenção, alcançando no final de 3 meses uma cobertura de 51,3\%. 
Em relação a comunidade, a participação e a aceitação do programa sobre saúde do idoso foi boa. Eles reconheceram o grande esforço que a equipe fez além da carência de recursos e infraestrutura. $\mathrm{O}$ apoio de segmentos locais como a diretora da escola do bairro que nos permitiu usar diferentes salas para realizar as atividades grupais, assim como todo o espaço físico da escola para nossas atividades. $\mathrm{O}$ apoio das universidades da cidade, também, foi muito importante pois contribuíram com a prestação de serviços gratuitos, na realização das atividades especialmente, no registro e busca ativa dos pacientes. Os estagiários foram acolhidos e incluídos nas atividades rotineiras da unidade.

Em relação a equipe e ao serviço em geral, obteve uma experiência muito gratificante, permitindo a integração de todos os membros da equipe e o trabalho simultâneo. Esse sentimento de parceria e solidariedade permitiu superar grandes dificuldades técnicas e materiais.

\section{REFERÊNCIAS}

1. A ONU e as pessoas idosas [Internet]. [cited 2019 Dec 13]. Available from: https:// nacoesunidas.org/acao/pessoas-idosas/

2. IBGE Projeção da população [Internet]. [cited 2019 Dec 13]. Available from: https://www.ibge.gov.br/apps/populacao/projecao/

3. Zaslavsky C, Gus I. Idoso. Doença cardíaca e comorbidades. Arq Bras Cardiol. 2002;79(6):635-9.

4. Martins RML, Santos ACA. Ser idoso hoje. Millenium. 2008; 35(13): 1-8.

5. Medeiros KKAS, Pinto Júnior EP, Bousquat A, Medina MG. O desafio da integralidade no cuidado ao idoso, no âmbito da Atenção Primária à Saúde. Saúde em Debate. 2017;41(spe3):288-95.

6. Santos SC, Tonhom SFR, Komatsu RS. Saúde do idoso: reflexões acerca da integralidade do cuidado. Rev Bras Promoção da Saúde. 2016;29(sup):118-27.

7. Galloza J, Castillo B, Micheo W. Benefits of Exercise in the Older Population. Phys Med Rehabil Clin N Am. 2017;28(4):659-69.

8. Varrelis MLZ. O paciente com necessidades especiais na odontologia: Manual prático. 3a ed. São Paulo:GEN Santos; 2017.

9. Presa SL; Matos J. Saúde bucal na terceira idade oral health in third age. Rev Uninga, Mar. 2014;39:137-48. 
10. Katrein F, Tejada CAO, Restrepo-Méndez MC, Bertoldi AD. Desigualdade no acesso a medicamentos para doenças crônicas em mulheres brasileiras TT. Cad Saude Publica. 2015;31(7):1416-26.

11. Bertoldi AD, Barros AJD, Camargo AL, Hallal PC, Vandoros S, Wagner A, et al. Household expenditures for medicines and the role of free medicines in the brazilian public health system. Am J Public Health. 2011;101(5):916-21.

12. Arrais PSD, Brito LL, Barreto ML, Coelho HLL. Prevalência e fatores determinantes do consumo de medicamentos no Município de Fortaleza, Ceará, Brasil. Cad Saude Publica. 2005 Dec;21(6):1737-46.

13. Aziz MM, Calvo MC, Schneider IJC, Xavier AJ, D’Orsi E. Prevalência e fatores associados ao acesso a medicamentos pela populaçáo idosa em uma capital do sul do Brasil: Um estudo de base populacional. Cad Saude Publica. 2011;27(10):1939-50.

14. Fernandes MTO. A Rede de Atenção à Saúde para o Cuidado à Pessoa Idosa na Atenção Primária: Um Modelo Teórico [Tese de doutorado]. Belo Horizonte: Universidade Federal de Minas Gerais; 2013.

15. Brasil. Ministério da Saúde. Secretaria de atenção à saúde. Departamento de atenção especializada e temática. Diretrizes para o cuidado das pessoas idosas no SUS: proposta de modelo de atenção integral. [Internet]. Brasília: MS; 2014 Acesso em 21 jan 2020. Disponível em: https://bvsms.saude.gov.br/bvs/publicacoes/diretrizes_ cuidado_pessoa_idosa_sus.pdf

16.Brasil. Ministério da Saúde. Portaria no 2528 de 19 de outubro de 2006. [Internet]. Acesso em: 21 jan 2020. Disponível em: https://bvsms.saude.gov.br/bvs/saudelegis/ gm/2006/prt2528_19_10_2006.html

Autor Correspondente: Diego Germán Ledesma E-mail: diegogermanleds_doctor@hotmail.com

Recebido em: 2020-01-14 Aprovado: 2020-04-28 